\title{
Factors that determine the Work Ability Index of street cleaners
}

\author{
Jung Won Kim ${ }^{1}$, Seonhee Yang ${ }^{1}$, Insung Chung ${ }^{1,2}$, Mi-Young Lee ${ }^{1,2}$ \\ ${ }^{1}$ Department of Occupational and Environment Medicine, Keimyung University Donsan Medical Center, Daegu, Korea \\ ${ }^{2}$ Department of Preventive Medicine, Keimyung University School of Medicine, Daegu, Korea
}

Received: October 30, 2018

Revised: April 11, 2019

Accepted: April 23, 2019

Corresponding author:

Mi-Young Lee

Department of Preventive Medicine,

Keimyung University School of

Medicine, 1095, Dalgubeol-daero,

Dalseo-gu, Daegu 42601, Korea

Tel: $+82-53-250-7874$

Fax: +82-53-250-7850

E-mail: deresa.lee2@gmail.com
Background: Korea has shown a rapid pace of aging and has become an aged society in a shorter period than Western countries. Therefore, it is important that middle-aged workers maintain their ability to work in the industrial field at the maximum level while maintaining their health. The purpose of this study was to analyze the factors affecting work ability and to use this data to maintain the working ability of middle-aged workers in the future.

Methods: From 2015 to 2017, we surveyed 371 street cleaners who visited a health center for health promotion in a metropolitan city. The relationship between the general characteristics of the survey subjects and the Work Ability Index (WAI) was confirmed by a univariate analysis. Statistically significant $(p<0.2)$ factors were selected and a multiple linear regression analysis was conducted with WAI scores as a dependent variable.

Results: Age, body mass index, body fat percentage, the Alcohol Use Disorders Identification Test-Korea (AUDIT-K) scores, the Psychosocial Well-Being Index-Short Form (PWI-SF) scores, maximal oxygen consumption $\left(\mathrm{VO}_{2 \max }\right)$, and hypertension showed statistically significant correlations with the WAI in the univariate analysis. In the multivariate analysis, the $\mathrm{VO}_{2 \text { max }}$ AUDIT-K scores, and PWI-SF scores were included in the regression equation. $\mathrm{VO}_{2 \max }$ had the greatest effect on the standardization coefficient indicating the relative contribution of independent variables, followed by the AUDIT-K scores and the PWI-SF scores.

Conclusion: Programs to increase workers' physical work ability and alleviate psychosocial stress and drinking habits should be developed and implemented in the future.

Keywords: Middle aged; Oxygen consumption; Physical endurance; Work ability evaluation

\section{Introduction}

According to the Korea National Statistical Office, most street cleaners are over 50 years old; the mean age of street cleaners is 58.4 years, which is higher than the average ages of other workers-for instance, 50.4 years for managers, 38.5 years for professional workers, 39.6 years for office workers, 45.5 years for service workers, 39.1 years for sales workers, and 44.0 years for agricultural workers [1]. As of August 2018, the labor force partici- pation rate of the elderly is $69.1 \%$, and the employment rate of the elderly is $66.9 \%$ [2]. It is therefore important that middle-aged workers be able to maintain their health in the industrial setting and exercise their maximum work ability.

Work ability is the ability to carry out a given job well and to maintain physical and mental health. In Europe, a number of studies have indicated that increasing age reduces working ability [3-5]. Decreases in the youth population due to aging and increases in the mean age of the workforce are associated with re- 
duced work productivity owing to reduced working ability [6]. As workers age, disability and illness increase due to reduced working ability and declining physical and mental abilities [7]. According to the U.S. Bureau of Labor Statistics, the number of major accidents among street cleaners in 2016 was 5.7 per 1,000 workers, with 439 being industrial accidents, making it the fourth most dangerous job in the US [8].

Working ability largely involves power of the muscles and respiratory system to determine how much work can be done in a given amount of time and how long one can last at the same strength. Therefore, we used maximal oxygen consumption $\left(\mathrm{VO}_{2 \max }\right)$ as an indicator of aerobic work ability. $\mathrm{VO}_{2 \max }$ is highly correlated with total endurance including cardiopulmonary ability, oxygen transport ability of the blood, and oxygen utilization ability of tissues [9].

In addition to age and aerobic work ability, work performance, body mass index, personal health status, and psychosocial stress have been reported to affect Work Ability Index (WAI) [10-13]. The relationship between age and WAI is controversial. While there are studies reporting negative correlations between age and WAI due to decreased physical ability, cognitive ability, and reaction rates in elderly people, there is also a study reporting a positive correlation owing to increasing work proficiency [10]. Additionally, there are studies reporting no correlation between age and WAI [13].

A few reports indicate other factors affecting the WAI besides psychosocial stress, age, work type, and aerobic work ability, and there are also a few regarding drinking habits and WAI. Therefore, it is important to understand the relationship between age and WAI and to identify other factors that affect WAI, including drinking habits.

The purpose of this study was to identify the factors affecting the WAIs of street cleaners and to use these data for the evaluation and management of working ability among middle-aged workers in the future.

\section{Materials and methods}

The study population comprised 411 street cleaners who visited occupational health facilities in Daegu from 2015 to 2017. Of these, 371 people were included in the final study population after excluding 28 cases including a small percentage of female cases, 10 cases of individuals with existing cerebral cardiovascular disease or pulmonary disease, and two cases of incomplete responses to the questionnaire.

Body fat and skeletal muscle percentage rate were measured using an Inbody 770 (InBody Korea [HQ], Seoul, Korea). The
Alcohol Use Disorders Identification Test-Korea (AUDIT-K) was conducted with scores ranging from 0 to 27 . Higher scores indicated a higher risk of a drinking habit. Drinking status was categorized as "low" if the score was below 11 and otherwise "high" [14]. The Psychosocial Well-Being Index-Short Form (PWI-SF) questionnaire was used to assess psychosocial stress on 4-point scale; each item was assigned a score of 0-1-2-3 and the scores were added up. On a scale of 0 to 54 , scores lower than 27 were classified as "low," meaning low stress, and higher scores were classified as "high" [15].

Work ability was assessed using the WAI questionnaire. The WAI questionnaire [16]: consists of seven items on physical, mental, and social competence. Each item has a range of 0 to 10 , and on item 1, current work ability is measured on a 10-point scale as compared to most active work ability. On item 2, physical and mental conditions related to job performance are measured on a 5-point scale. The weight for each item is given differently depending on whether the worker has a blue-collar or white-collar occupation. On item 3, a score is given based on the number of diseases diagnosed by a doctor ( 1 point for more than 5 diseases, 7 points for no disease). On item 4, the degree of occupational disability due to disease is measured on a 6-point scale. Item 5 is measured on a 5-point scale assessing the number of days of absence or illness, and item 6 is measured on a 7-point scale measuring the extent to which the respondent expects to be able to continue working after 2 years. On item 7, the mental health status of workers is further divided and the results are summed to obtain a score. The total score is evaluated from a minimum of 7 points to a maximum of 49 points.

The $\mathrm{VO}_{2 \max }$ was calculated using the Bruce equation (85.42-13.73 $\times$ sex-0.409 $\times$ age $[\mathrm{yr}])-3.24-0.114 \times$ weight $(\mathrm{kg})$.

In this study, an independent $\mathrm{t}$-test and a correlation test were performed to investigate the variables that affect WAI. The adjustment variables, which showed $p$-values $\leq 0.2$ in the univariate analysis, were included in the multiple logistic regression as independent variables. WAI was the dependent variable. All statistical analyses were performed using IBM SPSS version 25.0 (IBM Co., Armonk, NY, USA). This study was approved by the Institutional Review Board of Keimyung University Hospital (2019-03047-001).

\section{Results}

The ages of the 371 subjects ranged from 21 to 70 years, and the mean age was 48.6 years (Table 1 ). There was a statistically significant negative correlation between age and WAI (Table 2). As age increased, WAI tended to decrease (Table 3). 
Table 1. Characteristics of study subjects

\begin{tabular}{|c|c|}
\hline Variable & Subject \\
\hline Total number of subjects & 371 \\
\hline Age (yr) & $48.6 \pm 10.5$ \\
\hline$<50$ & $204(55.0)$ \\
\hline$\geq 50$ & $167(45.0)$ \\
\hline Height (m) & $1.7 \pm 0.1$ \\
\hline Weight (kg) & $71.7 \pm 10.7$ \\
\hline BMI & $24.7 \pm 3.1$ \\
\hline Percent body fat (\%) & $20.8 \pm 6.2$ \\
\hline Percent skeletal muscle (\%) & $31.8 \pm 4.4$ \\
\hline $\mathrm{VO}_{2 \max }$ & $40.4 \pm 4.1$ \\
\hline \multicolumn{2}{|l|}{ AUDIT-K } \\
\hline Low & $251(67.7)$ \\
\hline High & $120(32.3)$ \\
\hline \multicolumn{2}{|l|}{ PWI-SF } \\
\hline Low & $339(91.4)$ \\
\hline High & $32(8.6)$ \\
\hline \multicolumn{2}{|l|}{ Smoking } \\
\hline No or ex & $218(58.8)$ \\
\hline Current & $153(41.2)$ \\
\hline \multicolumn{2}{|l|}{ Medication } \\
\hline \multicolumn{2}{|l|}{ Hypertension } \\
\hline Yes & $27(7.3)$ \\
\hline No & $344(92.7)$ \\
\hline \multicolumn{2}{|l|}{ Diabetes mellitus } \\
\hline Yes & $17(4.6)$ \\
\hline No & $354(95.4)$ \\
\hline \multicolumn{2}{|l|}{ Hyperlipidemia } \\
\hline Yes & $13(3.5)$ \\
\hline No & $358(96.5)$ \\
\hline
\end{tabular}

Values are presented as mean \pm standard deviation or number (\%). $\mathrm{BMI}$, body mass index; $\mathrm{VO}_{2 \max }$ maximal oxygen consumption; AUDIT-K, Alcohol Use Disorders Identification Test-Korea; PWI-SF, Psychosocial Well-Being Index-Short form.

Mean height was $170.3 \mathrm{~cm}$ and mean weight was $71.7 \mathrm{~kg}$. There was no statistically significant correlation between height and WAI or between weight and WAI $(p=0.173, p=0.123)$. The mean body mass index (BMI) was 24.7, and there was a statistically significant negative correlation $(p=0.007)$ between BMI and WAI. The mean body fat percentage was $20.8 \%$. There was a statistically significant negative correlation $(p=0.001)$ between percent body fat and WAI. There was a statistically significant positive correlation $(p<0.001)$ between $\mathrm{VO}_{2 \max }$ and WAI. When comparing AUDIT-K scores results, the low group included 251 participants $(67.7 \%)$ while the high group included 120 $(32.3 \%)$; there was a statistically significance difference $(p<0.001)$. When comparing PWI-SF scores results, there was a
Table 2. Comparisons for WAI by categorical variables

\begin{tabular}{lcc}
\hline $\begin{array}{c}\text { Variable } \\
\text { AUDIT-K }\end{array}$ & WAl & $<0.001^{\mathrm{b})}$ \\
Low & & \\
High & $40.78 \pm 5.318$ & \\
PWI-SF & $38.63 \pm 4.993$ & $0.002^{\mathrm{b})}$ \\
Low & & \\
High & $40.34 \pm 5.270$ & \\
Smoking & $37.34 \pm 4.959$ & 0.781 \\
No or ex & & \\
Current & $40.15 \pm 5.168$ & \\
Medication & $39.99 \pm 5.511$ & $0.035^{\mathrm{b}}$ \\
Hypertension & & \\
Yes & & \\
No & $38.02 \pm 7.495$ & 0.728 \\
Diabetes mellitus & $40.25 \pm 5.073$ & \\
Yes & & \\
No & $39.65 \pm 3.892$ & 0.52 \\
Hyperlipidemia & $40.11 \pm 5.367$ & \\
Yes & $39.15 \pm 4.543$ & \\
No & $40.12 \pm 5.333$ &
\end{tabular}

Values are presented as mean \pm standard deviation.

WAI, Work Ability Index; AUDIT-K, Alcohol Use Disorders Identification Test-Korea; PWI-SF, Psychosocial Well-Being Index-Short form.

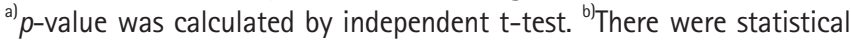
significance in WAI between group 1 and group $2(p<0.05)$.

statistically significant difference $(p=0.002)$ with the low group including 339 (91.4\%) participants, while the high group included 32 (8.6\%). Additionally, 27 cases (7.3\%) were taking antihypertensive medication, and there was a statistically significant difference in WAI scores between the antihypertensive medication group and the non-medication group $(p=0.035)$. In addition, there was no statistically significant difference between the two groups in terms of skeletal muscle percentage, smoking status, diabetes medication history, and hyperlipidemia medication history (Table 4).

The adjustment variables included in the multiple linear regression analysis were age, BMI, percent body fat, AUDIT-K scores, PWI-SF scores, and hypertension; these were included in the analysis because the $p$-values were $\leq 0.2$. In the stepwise linear multiple regression analysis with WAI and seven independent variables, $\mathrm{VO}_{2 \max }$, AUDIT-K scores, and PWI-SF scores were significant at a significance level of 0.05 ; age, $\mathrm{BMI}$, and body fat percentage were not significant. The $\mathrm{VO}_{2 \max }$ AUDIT-K scores, and PWI-SF scores accounted for $11.8 \%$ of the total variation. $\mathrm{VO}_{2 \max }$ had the greatest effect, followed by the AUDIT-K scores and PWI-SF scores results (Table 5). 
Table 3. Mean WAI in different age groups

\begin{tabular}{lccccccc}
\hline Age (yr) & $20-29$ & $30-34$ & $35-39$ & $40-44$ & $45-50$ & $50-55$ & over 55 \\
\hline Number & 6 & 23 & 61 & 56 & 58 & 45 & 122 \\
Mean & 43.67 & 42.33 & 41.02 & 40.20 & 40.51 & 40.76 & 38.52 \\
SD & 4.92 & 4.75 & 5.11 & 5.18 & 4.47 & 4.30 & 5.91 \\
\hline
\end{tabular}

WAI, Work Ability Index; SD, standard deviation.

Table 4. Correlations among WAI and other independent variables

\begin{tabular}{|c|c|c|c|c|c|c|c|c|}
\hline & Age (yr) & Height (m) & Weight (kg) & $\mathrm{BMI}$ & $\begin{array}{l}\text { Percent body } \\
\text { fat }(\%)\end{array}$ & $\begin{array}{l}\text { Percent skeletal } \\
\text { muscle }(\%)\end{array}$ & $\mathrm{VO}_{2 \max }$ & WAI \\
\hline Age (yr) & 1.000 & & & & & & & \\
\hline Height (m) & $-0.557^{\mathrm{a})}$ & 1.000 & & & & & & \\
\hline Weight $(\mathrm{kg})$ & $-0.345^{\mathrm{a})}$ & $0.542^{\mathrm{a})}$ & 1.000 & & & & & \\
\hline $\mathrm{BMI}$ & -0.061 & 0.013 & $0.845^{\mathrm{a})}$ & 1.000 & & & & \\
\hline Percent body fat (\%) & $0.292^{\mathrm{a})}$ & $-0.250^{a)}$ & $0.487^{\mathrm{a})}$ & $0.739^{a)}$ & 1.000 & & & \\
\hline Percent skeletal muscle $(\%)$ & $-0.594^{\text {a) }}$ & $0.764^{\mathrm{a})}$ & $0.782^{\mathrm{a})}$ & $0.442^{\mathrm{a})}$ & -0.081 & 1.000 & & \\
\hline $\mathrm{VO}_{2 \max }$ & $-0.960^{\text {a) }}$ & $0.429^{\text {a) }}$ & 0.066 & $-0.189^{a)}$ & $-0.457^{a)}$ & $0.397^{\mathrm{a})}$ & 1.000 & \\
\hline WAI & $-0.213^{\text {a) }}$ & 0.071 & -0.020 & $-0.141^{a)}$ & $-0.176^{a)}$ & 0.043 & $0.251^{\text {a) }}$ & 1.000 \\
\hline
\end{tabular}

Values are presented as Pearson's correlation coefficient.

WAI, Work Ability Index; $\mathrm{BMI}$, body mass index; $\mathrm{VO}_{2 \max }$ maximal oxygen consumption.

a) $p<0.05$.

Table 5. Relationship between WAI and AUDIT-K, PWI-SF and $\mathrm{VO}_{2 \max }$

\begin{tabular}{lccc}
\hline & $\beta$ & $p$-value & $\Delta R^{2}$ \\
VO $_{2 \max }$ & 0.239 & $<0.001$ & 6.3 \\
AUDIT-K & -0.178 & $<0.001$ & 3.2 \\
PWI-SF & -0.151 & 0.002 & 2.3 \\
& $R^{2}=11.8$ & & \\
\hline
\end{tabular}

Age, body mass index, percent body fat, taking antihypertension drugs were excluded.

WAI, Work Ability Index; $\mathrm{VO}_{2 \text { max }}$ maximal oxygen consumption; AUDIT-K, Alcohol Use Disorders Identification Test-Korea; PWI-SF, Psychosocial WellBeing Index-Short form.

a) $p$-value was calculated by multiple linear regression analysis.

\section{Discussion}

In this study, WAI was used to evaluate working ability. WAI was developed at the Finnish Institute of Occupational Health in Finland in 1994 for the purpose of assessing the working ability of older workers [16]. Factors influencing WAI include lack of leisure time, excessive workload, musculoskeletal abilities, age, obesity, lack of autonomy, and need of high physical abilities [17].

There has been much research on the relationship between age and WAI, but the findings are contradictory. Lee and Chang [10] reported that working ability and age were not linear; working ability seemed high in the early 20 s and 30 s age ranges but gradually decreased from the late 30s, and it was highest above 55 years due to proficiency. However, Bridger and Bennett [11] and Bugajska et al. [18] reported negative correlations. In this study, age and WAI seemed to be negatively correlated, which means that WAI decreases as age increases. Studies have reported negative correlations between age and WAI due to decreased physical ability, cognitive ability, and reaction rates among elderly people. However, other studies have reported positive correlations due to increased work proficiency.

In the present study, $\mathrm{VO}_{2 \max }$ was the most influential factor for WAI. In the regression equation of this study, the standardization factor of $\mathrm{VO}_{2 \max }$ was 0.239; as $\mathrm{VO}_{2 \max }$ increased, WAI also increased. As aerobic work ability increased, working ability also increased. Bugajska et al. [18] reported a positive correlation between $\mathrm{VO}_{2 \max }$ and WAI among 524 Polish women and 664 Polish male workers. However, Habibi et al. [19] reported no statistical significance between the two variables among 228 Iranian nurses. As working ability is affected by physical conditions, the higher the $\mathrm{VO}_{2 \max }$, which indicates aerobic work ability, the greater the working ability. In particular, street cleaners show a large 
correlation between $\mathrm{VO}_{2 \max }$ and WAI because they engage in many outdoor activities.

The standardization factor of the AUDIT-K scores was -0.178 , and the standardization factor of the PWI-SF scores was -0.151 . In this study, the groups with good drinking habits and low psychosocial stress had great working ability. Hur et al. [15] reported that there was no statistically significant relationship between drinking habits and work ability, but reported that working ability decreased with increasing psychosocial stress. Ye et al. [20] reported that the higher the level of psychosocial stress, the lower the working ability. Kim and Kim [13] and Lee et al. [14] also reported that stress and WAI were negatively correlated. It is suggested that stress management is helpful for improving work ability. The relationship between drinking habits and working ability were unknown in previous studies. In the present study, the group with good drinking habits showed higher WAI. Alcohol weakens attentiveness, motor coordination abilities, and response speed to external stimuli; thus, post-drinking tasks show reduced job accuracy and efficiency. There is also the possibility of reducing working ability by increasing impulsive behavior and weakening temperance. Therefore, proper drinking habits should improve work ability.

In this study we used the WAI, which can visually evaluate the abstract concept of working ability, to increase the reliability of the study. Age and also body fat percentage, alcohol status, and psychosocial stress were included as independent factors.

The present study has several limitations. It is difficult to generalize the findings because it investigated mostly male street cleaners in only one area. Factors such as job stress or shift work may also affect work ability, but these factors are not reflected. In addition, there is a limitation in that $\mathrm{VO}_{2 \max }$ cannot be obtained directly but was estimated using an indirect formula.

The significant findings of this study showed that aerobic physical ability, drinking habits, and psychosocial stress were correlated with working ability. Experimental evaluation of working ability, such as oxygen consumption and maximum heart rate, has the advantage of obtaining accurate results under constrained conditions, but it is difficult to apply at the worksite, and there are also time and cost constraints. On the other hand, work performance evaluation using the WAI questionnaire is subjective. Reliability is not high compared with the experiment, but this evaluation method is simple, time and cost effective, and easy to apply at the actual work site. Therefore, middle-aged workers will be able to utilize WAI as an indicator of their working ability in programs that can then easily evaluate and maintain their physical ability.

\section{ORCID}

Jung Won Kim, https://orcid.org/0000-0002-7090-5687

Seon Hee Yang, https://orcid.org/0000-0002-2487-1324

In Sung Jung, https://orcid.org/0000-0001-8532-1070

Mi Young Lee, https://orcid.org/0000-0002-2210-3890

\section{Conflicts of interest}

No potential conflicts of interest relevant to this article was reported.

\section{References}

1. Statistics Korea. [Job, gender, salary and working conditions] [Internet]. Daejeon: Statistics Korea; 2018 [cited 2018 October 5]. http: / / kosis.kr/statHtml/statHtml.do?orgId = 118\&tbIId=DT_118N_PAYM31\&conn_path = I2

2. Statistics Korea. [Employment trends for the elderly] [Internet]. Daejeon: Statistics Korea; 2018 [cited 2018 October 5]. http:// www.index.go.kr/potal/main/EachDtlPageDetail.do?idx_ $\mathrm{cd}=1496$

3. Ilmarinen J, Tuomi K, Klockars M. Changes in the work ability of active employees over an 11-year period. Scand J Work Environ Health 1997;23(Suppl 1):49-57.

4. Tuomi K, Huuhtanen P, Nykyri E, Ilmarinen J. Promotion of work ability, the quality of work and retirement. Occup Med (Lond) 2001;51:318-24.

5. Liira J, Matikainen E, Leino-Arjas P, Malmivaara A, Mutanen P, Rytkönen H, et al. Work ability of middle-aged Finnish construction workers - a follow-up study in 1991-1995. Int J Ind Ergon 2000;25:477-81.

6. de Zwart BC, Frings-Dresen MH, van Dijk FJ. Physical workload and the aging worker: a review of the literature. Int Arch Occup Environ Health 1995;68:1-12.

7. Kim YU, Kim JG. The evaluation of work ability and job stress for subway worker.J Korean Soc Saf 2008;23:85-9.

8. U.S. Bureau of Labor Statistics. 2016 Census of Fatal Occupational Injuries (final data) [Internet]. Washinton, DC: U.S. Bureau of Labor Statistics; 2017 [cited 2018 October 5]. https://www.bls. gov/iif/oshcfoil.htm\#2016

9. Wagner PD. New ideas on limitations to VO2max. Exerc Sport Sci Rev 2000;28:10-4.

10. Lee YJ, Chang SR. A study on the Work Ability Index by the type of business, age and job. J Ergon Soc Korea 2010;29:101-5.

11. Bridger RS, Bennett AI. Age and BMI interact to determine work ability in seafarers. Occup Med (Lond) 2011;61:157-62. 
12. Lee YJ, Chang SR. A study on the job stress and the work ability of workers in the shipbuilding industry. J Korean Soc Saf 2010;25:71-7.

13. Kim YC, Kim JK. A study on evaluation of work ability and job stress for doctor.J Ergon Soc Korea 2008;27:9-14.

14. Lee BO, Lee CH, Lee PG, Choi MJ, Namkoong K. Development of Korean version of Alcohol Use Disorder Identification Test (AUDIT-K): its reliability and validity.J Korean Acad Addict Psychiatry 2000;4:83-92.

15. Hur SO, Chang SS, Koo JW, Park CY. The assessment of stress between white and blue collar workers by using Psychosocial Well-being Index. Korean J Prev Med 1996;29:609-16.

16. Ilmarinen J. The work ability index (WAI). Occup Med 2007;57:160.
17. van den Berg TI, Elders LA, de Zwart BC, Burdorf A. The effects of work-related and individual factors on the Work Ability Index: a systematic review. Occup Environ Med 2009;66:211-20.

18. Bugajska J, Makowiec-Dąbrowska T, Jegier A, Marszałek A. Physical work capacity (VO2 max) and work ability (WAI) of active employees (men and women) in Poland. Int Congr Ser 2005; 1280:156-60.

19. Habibi E, Dehghan H, Zeinodini M, Yousefi H, Hasanzadeh A. A study on Work Ability Index and physical work capacity on the base of fax equation $\mathrm{VO}(2)$ max in male nursing hospital staff in Isfahan, Iran. Int J Prev Med 2012;3:776-82.

20. Ye S, Kang D, Kim Y, Son M, Kim J, Park S. The relationship between work ability and job stress factors in manufacturing industries. Ann Occup Environ Med 2008;20:260-71. 